\title{
Erratum
}

\section{Synthesis of Bicyclic Ureas via Palladium-Catalyzed Carboamination}

N. R. Bbaij, J. P. Wolfe* Synfacts 2013, 9, 1192.

In 9-epi-batzelladine $\mathrm{K}$ and the immediate precursor directly to the left (the substrate for the $\mathrm{NaBH}_{3} \mathrm{CN}$ reduction) one $\mathrm{NH}$ group was depicted as an NAr group. We apologize for this mistake. The correct structures are shown below.<smiles></smiles>
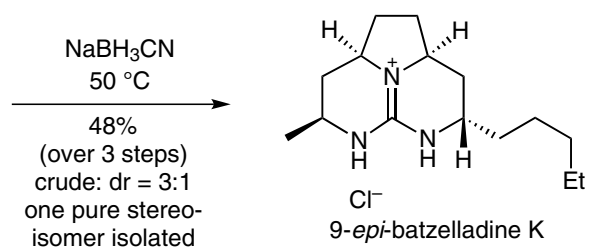